\title{
Endoscopic management of a migrated biliary T-tube
}

The accidental migration of a biliary $\mathrm{T}$ tube is a rare complication. A 38-year-old man presented with jaundice and abdominal pain. He had undergone open cholecystectomy and common bile duct (CBD) exploration 1 month previously for cholelithiasis and choledocholithiasis, with a Ttube placed in the CBD during surgery for subsequent biliary decompression. On examination, he was markedly icteric and had right upper quadrant tenderness. It was noted that the external end of the Ttube had accidentally migrated into peritoneal cavity. Laboratory investigations were as follows: total bilirubin $6.4 \mathrm{mg} / \mathrm{dL}$, aspartate transaminase (AST) $62 \mathrm{U} / \mathrm{L}$, alanine transaminase (ALT) $110 \mathrm{U} / \mathrm{L}$, serum alkaline phosphatase (ALP) $224 \mathrm{U} / \mathrm{L}$. Plain radiographs and ultrasound examination of the abdomen revealed that the whole of the T-tube was within the peritoneal cavity.

Endoscopic retrograde cholangiopancreatography (ERCP) was performed after the patient had received initial treatment with prophylactic antibiotics. A stricture was observed in the lower CBD, which was dilated using a $10-\mathrm{mm}$ biliary balloon. The T-tube had a radio-opaque marker, but this was absent because of guttering of the tube at the time of deployment; hence the T-tube was not visible inside the $\mathrm{CBD}$ fluoroscopically ( Fig. 1). An endoscopic sphincterotomy was performed. The end of the T-tube arm nearest to the sphincter was grasped with foreign-body grasping forceps and the whole $50-\mathrm{cm}$ length of the T-tube was extracted ( $\bullet$ Fig. 2). Following the procedure the patient improved and had no complaints during follow-up for 1 year. The complications of T-tube retrieval include bile leakage, peritonitis, bacteremia, retention of a fragment, and stenosis [1]. There are a few reports of patients with complications from retained remnant $\mathrm{T}$ tube fragments [2-5]. To the best of our knowledge, this is the first report of the whole length of a T-tube having migrated into the peritoneal cavity and its successful endoscopic management. The technique applied in this case, which is simple and feasible, provided a method for the endoscopic removal of a T-tube that had undergone intraperitoneal migration,

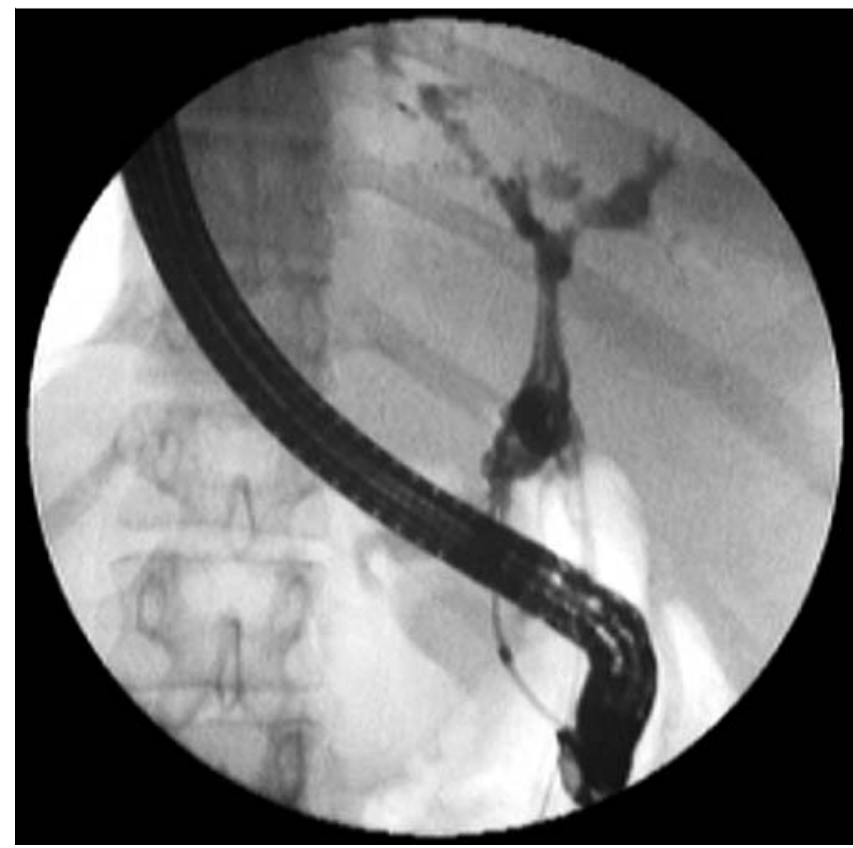

Fig. 1 Fluoroscopic image during endoscopic retrograde cholangiopancreatography (ERCP) showing the presence of the migrated T-tube.

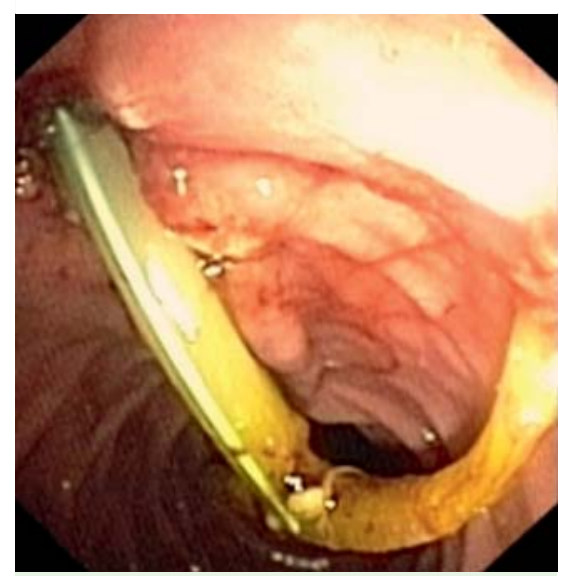

Fig. 2 Endoscopic view showing retrieval of the T-tube.

using a foreign-body forceps. Migrated Ttubes should be retrieved immediately to avoid both early and late complications. Surgical retrieval of a migrated, whole biliary T-tube is not required and can be replaced by this endoscopic method.

\section{Endoscopy_UCTN_Code_CPL_1AK_2AI}

Competing interests: None

\section{A. Krishnan, R. Ramakrishnan}

Department of Gastroenterology, Apollo Hospitals, Chennai, India
References

1 Wills VL, Gibson K, Karihaloot C, Jorgensen JO. Complications of biliary T-tubes after choledochotomy. ANZ J Surg 2002; 72: 177 180

2 Mapelli P, Veiga A, Monticciolo R. Retained Ttube fragment: Removal using ERCP with papillotomy. Am J Gastroenterol 1988; 83: 1005

3 Muhammad SR, Gatehouse D. Removal of a retained T-tube from the common bile duct. J Pak Med Assoc 1997; 47: 194-195

4 McCarthy MC, Becker GJ, Hegyi GJ. Retained T-tube fragment: Removal using a Gruntzig balloon dilatation catheter. Indiana Med 1986; 79: $772-773$

5 Shamimi K, Moazami F, Jalali M, Aminian A. Delayed cholangitis due to a retained $\mathrm{T}$ tube fragment. Ind J Surg 2006; 68: 328 329

Bibliography

DOI $10.1055 / \mathrm{s}-0030-1256739$

Endoscopy 2011; 43: E325

(c) Georg Thieme Verlag KG Stuttgart · New York . ISSN 0013-726X

Corresponding author

A. Krishnan, MBBS

C-137, Karthikeyan salai

Periyar Nagar, Perambur

Chennai-600083

India

dr.arunkumarpillai@gmail.com 